\title{
Wissens- und lernorientierte Instandhaltung unter dem Aspekt zunehmender Digitalisierung
}

\author{
Cornelia Huber (D) - Theresa Passath • Hubert Biedermann
}

Eingegangen: 22. Juni 2020 / Angenommen: 5. Oktober 2020 / Online publiziert: 14. Oktober 2020 (C) Der/die Autor(en) 2020

Zusammenfassung Die Digitalisierung bietet Industrieunternehmen eine Vielzahl von Möglichkeiten und Lösungen (z. B. Big Data Analytics, Cloud-Computing, Internet of Things), die mit zunehmenden Anforderungen an das Wissensmanagement verbunden sind. Ein angepasstes Assetmanagement zur Sicherstellung von Markterfolgsfaktoren sind für die Smart Factory unerlässlich. Ein ganzheitliches wertschöpfungsorientiertes Managementkonzept bietet die Lean Smart Maintenance Philosophie, das den geänderten Umfeldbedingungen Rechnung tragend, eine dynamisch anpassbare Instandhaltungsstrategie ermöglicht. Insbesondere die Ressource Wissen gewinnt in Anbetracht einer ständig steigenden Anlagenkomplexität in Form der wissen- und lernorientierten Instandhaltung an Bedeutung, damit zukünftig der Einsatz neuer Technologien nachhaltig wirtschaftlich ist. In diesem Zusammenhang wird nachstehend eine praxisnahe Vorgehensmethodik präsentiert, die die Externalisierung impliziten Wissens durch die Kombination der Wissensbausteine nach Probst mit dem DMAIC-Zyklus unterstützt. Die Digitalisierung induziert geänderte Anforderungen an die Mitarbeiter und Mitarbeiterinnen; geeignete Qualifizierungsmaßnahmen sollten für die bestmögliche Weiterentwicklung der Humanressourcen frühzeitig erkannt und implementiert werden.

Schlüsselwörter Wissensmanagement · Digitalisierung · Lean Smart Maintenance · Qualifizierung · DMAIC · Asset Management

C. Huber $(\bowtie) \cdot$ T. Passath $\cdot$ H. Biedermann

Lehrstuhl für Wirtschafts- und Betriebswissenschaften, Montanuniversität Leoben,

Peter-Tunner-Straße 25-27, 8700 Leoben, Österreich

E-Mail: cornelia.huber@unileoben.ac.at 


\section{Learn-oriented Maintenance in the Age of Digitalisation}

Abstract Digitalization offers industrial companies a multitude of possibilities and solutions (e.g. Big Data Analytics, Cloud Computing, Internet of Things), which are associated with increasing demands on knowledge management. An adapted asset management is essential for Smart Factory to ensure market success factors. The Lean Smart Maintenance philosophy offers a holistic value-added oriented management concept that considers the changed environmental conditions and enables a dynamically adaptable maintenance strategy. In view of the ever-increasing complexity of assets, knowledge as a resource is becoming increasingly important for a knowledge- and learning-oriented maintenance, so that the use of new technologies will be sustainable economical in the future. In this context, a practice-oriented process methodology is presented below that supports the externalization of implicit knowledge by combining the Probst knowledge modules with the DMAIC cycle. Digitization induces changed demands on employees; suitable qualification measures should be recognized and implemented at an early stage to guarantee the best further development of human resources.

Keywords Lean smart maintenance - Knowledge management · Digitalization · Qualification · DMAIC · Asset management

\section{Einleitung}

Die digitale Transformation und die damit verbundene horizontale und vertikale Datenintegration haben über die Supply Chain in sämtlichen Bereichen eines Unternehmens einen wesentlichen Einfluss auf die Arbeit der Zukunft. Durch diese Veränderungen in der Arbeitswelt, sowie den Qualifizierungsanforderungen ist es wichtig, das Thema Wissensmanagement in Unternehmen zu forcieren, um der Gefahr eines Wissens- und dem damit einhergehenden Kompetenzverlustes entgegenzuwirken.

Wissensmanagement umfasst zahlreiche Methoden und Modelle zur Erfassung, Bewertung, Verteilung, Anwendung und Bewahrung des Wissens einer Organisation. Aktuell ist in zahlreichen Betrieben Verbesserungspotenzial gegeben, wie eine Benchmarkstudie auf diesem Gebiet zeigt. Von den 188 befragten Unternehmen der DACH-Region verschiedenster Branchen, haben weniger als 50\% einen standardisierten Prozess zum Wissenserhalt und der -weitergabe definiert. Des Weiteren haben erst 39\% der Befragten eine Wissensdatenbank implementiert (vgl. Biedermann 2018).

Nachfolgend wird ein adäquates Wissensmanagement als wesentlicher Baustein der Lean Smart Maintenance Philosophie näher beschrieben. In dem Artikel wird adressiert, wie nachhaltige Wissenssicherung durch den Einsatz von Informationstechnologie und die Einführung eines Condition Monitoring Systems für eine permanente Analgenüberwachung realisiert werden kann. 


\section{Integration von Wissensmanagement im Lean Smart Maintenance (LSM) Konzept}

Eine effiziente und effektive Implementierung von Wissensmanagement in der Instandhaltung $(\mathrm{IH})$ ist anhand einer konzeptiven Berücksichtigung der drei Managementebenen normativ, strategisch und operativ möglich (vgl. Passath und Huber 2019, S. 7). Die Lean Smart Maintenance (LSM) Philosophie ist ein Konzept, das auf Regelkreisen (Lernschleifen) beruht und zur dynamischen Prozessverbesserung führt. Dabei liegt der Fokus des Lean-Bereiches auf der schlanken Instandhaltung zur Effizienzsteigerung durch kontinuierliche Prozessoptimierung. Der Smart-Bereich adressiert die lern- und wissensorientierte Instandhaltung zur Effektivitätssteigerung durch dynamische Anpassung der Instandhaltungsstrategie (vgl. Biedermann 2016). Ein Ziel der LSM-Philosophie ist die Quantifizierung des Wertschöpfungsbeitrags. Durch die Betrachtung des gesamten Lebenszyklus der Anlagen, soll eine maximale Anlagenzuverlässigkeit zu minimalen Kosten erreicht werden. Ein wesentlicher Teil für dessen Umsetzung bildet das Wissensmanagement, das alle Bereiche, Ebenen und Teilfunktionen der Organisation umfasst (vgl. Biedermann 2016).

Wissensmanagement hat in diesem ganzheitlichen Ansatz einen wesentlichen Einfluss auf die Bereichskultur und die Funktionen, Ziele, Organisation, Strategieplanung und die kontinuierliche Verbesserung (KVP). Dabei bildet Wissensmanagement durch die Aufbereitung, Darstellung, Verwaltung und Weitergabe von Daten und den daraus gewonnenen Informationen die Grundlage, um den Wissenserwerb zu erleichtern, langfristig zu sichern und Unternehmensziele dauerhaft zu erfüllen. Dieses Ziel wird durch den Einsatz verschiedener Methoden und Instrumente des LSM-Konzeptes, wie etwa einer dynamischen/einheitlichen Anlagenbewertung erreicht. Diese greift ebenfalls auf das Wissen der Organisation und der Mitarbeiter und Mitarbeiterinnen zurück, wodurch eine Instandhaltungsoptimierung im Bereich der Strategie und/oder der Prozesse ermöglicht wird (vgl. Biedermann 2016).

\subsection{Zusammenspiel von Mensch, Organisation und Technik}

Das Wissensmanagement, als Teil der wertschöpfungsorientierten LSM-Philosophie, beinhaltet als ganzheitlicher Ansatz die Aspekte Mensch, Organisation und Technik (MOT). Dieser Ansatz nach Bullinger et al. (1998) ist in Kombination mit dem Strukturmodell des intellektuellen Kapitals zu betrachten.

Der Schwerpunkt im Bereich Mensch liegt auf der Gestaltung der Organisationskultur, der kontinuierlichen Unterstützung des Wissensflusses, der Motivation und Kommunikation. Der Mensch hat die Funktion des Wissensträgers, der das soziale System Organisation und Unternehmen begründet und somit das Humankapital (HK) abbildet (vgl. Biedermann 2003, S. 488). Im Bereich Organisation werden Methoden für Wissenserwerb, -speicherung und -transfer, sowie die Strukturen, Zuständigkeiten und Prozesse fokussiert. Die Vernetzung der Wissensträger: Mitarbeiter, Lieferanten, Kunden, Kooperationspartner etc., durch die Organisation wird über das Beziehungskapital (BK) abgebildet (vgl. Biedermann 2003, S. 488). Im dritten Bereich Technik liegt der Schwerpunkt auf der Informations- und Kommunikationstechnologie, Datenbanken, Softwarelösungen, Schnittstellen zwischen 
Mensch-Maschine und/oder Softwaresystemen, der durch die Digitalisierung an Bedeutung gewinnt. Die Technik stellt die notwendige Infrastruktur für die Gestaltung der Wertschöpfungsprozesse bereit und in Kombination mit der Organisation wird das Strukturkapital (SK) messbar (vgl. Biedermann 2003, S. 488).

Das Zusammenwirken des Strukturmodells mit dem MOT-Modell ist möglich, da die Inhalte dieses Modells Ähnlichkeiten zu den Rahmenbedingungen für Humankapital (HK), Strukturkapital (SK) und Beziehungskapital (BK), wie z. B. Motivation, Wandlungsbereitschaft, Prozesse, Organisationsstrukturen, Technologieeinsatz, IHSteuerung und Durchführung aufweisen. HK, SK und BK konstituieren das intellektuelle Kapital und werden in einer Wissensbilanz abgebildet, in der diese als Inputfaktoren für die Leistungsprozesse dienen (vgl. Biedermann 2003). Durch die Zusammenführung des MOT-Modells mit dem Strukturmodell ist es möglich nichtmonetäre Größen anhand von Kennzahlen zu messen.

Interessant in diesem Modell sind die Wechselwirkungen der Faktoren (Mensch, Organisation, Technik), wobei eine stetige Verbindung der kontinuierlich involvierten Kapitalarten (HK, SK, BK) notwendig ist. In Kombination mit den Markterfolgsfaktoren Kosten, Zeit und Qualität (vgl. Seghezzi et al. 2007, S. 29) tragen diese zu einer langfristigen, wissensbasierten, wertschöpfungsorientierten Unternehmensführung bei.

Das Kriterium Qualität auf der Technikebene in Bezug auf Informations- und Kommunikationstechnologie konkretisiert die vom Menschen wahrgenommenen Eigenschaften wie etwa „Systemverfügbarkeit“, „Funktionalität“ und „Leistungserbringung“. Die Humanebene beinhaltet Qualitätsmerkmale wie z. B. „Instandhaltungs- und Wissenszielerreichung“, „Mitarbeiterintegration“, „Beziehungsqualität“" (vgl. Nissen et al. 2018, S. 815). Der Qualitätsbezug zur Organisation kann die Elemente „Wissensqualitätslevel“ und „Entwicklungszyklen“ enthalten. Ein langfristiger Zeithorizont hat einen positiven Einfluss auf das MOT-Modell und das Organisationswissen, indem das Vertrauen der Mitarbeiter und Mitarbeiterinnen gefestigt, dadurch die Bereitschaft zum Wissensaustausch erhöht, die Reziprozität gesteigert und die internen Prozesse fokussiert werden.

Ein kurzfristiger Zeithorizont erhöht die Reaktionszeit und Flexibilität der Instandhaltung und der Fokus wird auf die Kunden- und Marktorientierung gerichtet. Somit sind interne (Wissens-) Prozesse nachgelagert und eine Implementierung von Wissensmanagementprozessen hat keine hohe Relevanz. Die Generierung von neuem Wissen in der Instandhaltung steht im Vordergrund, das durch externen Wissensaustausch bestärkt wird (schnelle Marktreaktion). Das Kriterium Kosten wird mit dem Ressourceneinsatz verbunden, welcher auf der Technikseite mitunter den Bereich der Anschaffung und die Anwendung von Informations- und Kommunikationstechnologien beeinflusst (vgl. Nissen et al. 2018).

Allerdings besteht die Möglichkeit, dass die Markterfolgsfaktoren Kosten, Zeit und Qualität konkurrieren und dadurch Zielkonflikte entstehen. Um dem entgegenzuwirken, müssen diese durch eine optimale Geschäftsprozess- und Wissensmanagementplanung frühzeitig aufeinander abgestimmt werden (vgl. Schneider et al. 2008, S. 55f). Die Kombination des Qualitätsdreiecks mit dem MOT-Modell und zusätzlich den Kapitalarten des Wissens führt zu einem Mehrwert im Bereich Qualitätssicherung von Wissensprozessen und damit einhergehend zu einer Steigerung 
der Motivation und Akzeptanz der Mitarbeiter und Mitarbeiterinnen durch erhöhte Prozesstransparenz.

Durch neue Technologien und steigenden Digitalisierungsgrad ist es erforderlich, die Zusammenarbeit zwischen IT und Instandhaltung zur Wertschöpfungssteigerung zu stärken. Der frühzeitige Kompetenz- und Wissensabgleich beider Bereiche im Hinblick auf neue Technologien und deren Anwendungen schafft einen strategischen Mehrwert für Industriebetriebe (vgl. Urbach und Ahlemann 2017). Die Kooperation und der kontinuierliche Informationsaustausch einzelner Fachbereiche z. B. Produktion, Instandhaltung, F\&E, IT innerhalb eines Unternehmens bilden eine solide Basis für ein funktionierendes Wissensmanagement. Hierzu ist der Einsatz von Technologien (Intranet, Datenbanken, Kollaborationsinstrumente etc.) wesentlich.

\section{Wissen- und lernorientierte Instandhaltung}

Die normative Managementebene, deren Schwerpunkt auf dem Humankapital liegt, beinhaltet die Unternehmenskultur, Instandhaltungs- und Wissensziele und das Leitbild. Die organisatorische Instandhaltungs-Struktur sowie die Wissens- und Instandhaltungs-Strategie sind auf der strategischen Ebene enthalten, ebenso wie Problemlösungskompetenzen, wodurch der Fokus auf das Beziehungskapital gelegt wird. Das Kernwissen und das Fähigkeitsportfolio (Q-Matrix) ist der strategischen Ebene zuzurechnen, wodurch das Humankapital auf dieser Ebene integriert wird. Auf der operativen Ebene werden die Instandhaltungs- und Wissensplanungsprozesse betrachtet, sowie die Umsetzung der strategischen Instandhaltungsvorgaben und die Optimierung der Wissensinfrastruktur. Auch das Leistungs- und Kooperationsverhalten der Instandhaltung und der Stakeholder, die Optimierung der InstandhaltungsWissensstruktur und das Shopfloormanagement werden auf der operativen Ebene berücksichtigt, wodurch verstärkt das Strukturkapital betrachtet wird (Abb. 1; vgl. Probst et al. 2006).

Ziel ist es Instrumente des Wissensmanagement vertikal, über alle drei Managementebenen hinweg und horizontal über die gesamte Wertschöpfungskette eines Unternehmens dauerhaft zu integrieren. Für die Implementierung der Wissensmanagementinstrumente ist die Anwendung des DMAIC-Zyklus zu empfehlen. Im Bereich Wissensmanagement gibt es eine Vielzahl an Modellen und Methoden, die per se unterschiedliche Wissensmanagementinstrumente zur Verfügung stellen. Bekannte Wissensmanagementmodelle sind unter anderem die Wissenstreppe von North et al. (2016), das Bausteinmodell von Probst et al. (2006), das Potsdamer Modell von Gronau (2009) oder das SECI-Modell von Nanoka und Takeuchi (1995). Durch Gegenüberstellung der einzelnen Modelle konnten 4-Phasen, beginnend mit der Identifikation, Erwerb \& Entwicklung, Verteilung \& Nutzung, bis hin zur Bewertung des Wissens, abgeleitet werden. Für die Definition und Zuteilung der Wissensmanagementinstrumente mit der Anwendbarkeit in der Instandhaltung wurden ausgewählte Wissensmanagementmethoden genauer analysiert. Aufgrund der Heterogenität der Wissensmanagementinstrumente gibt es verschiedene Klassifizierungen in der Literatur. Lehner (2006) unterscheidet zwischen Wissensaustausch \& 


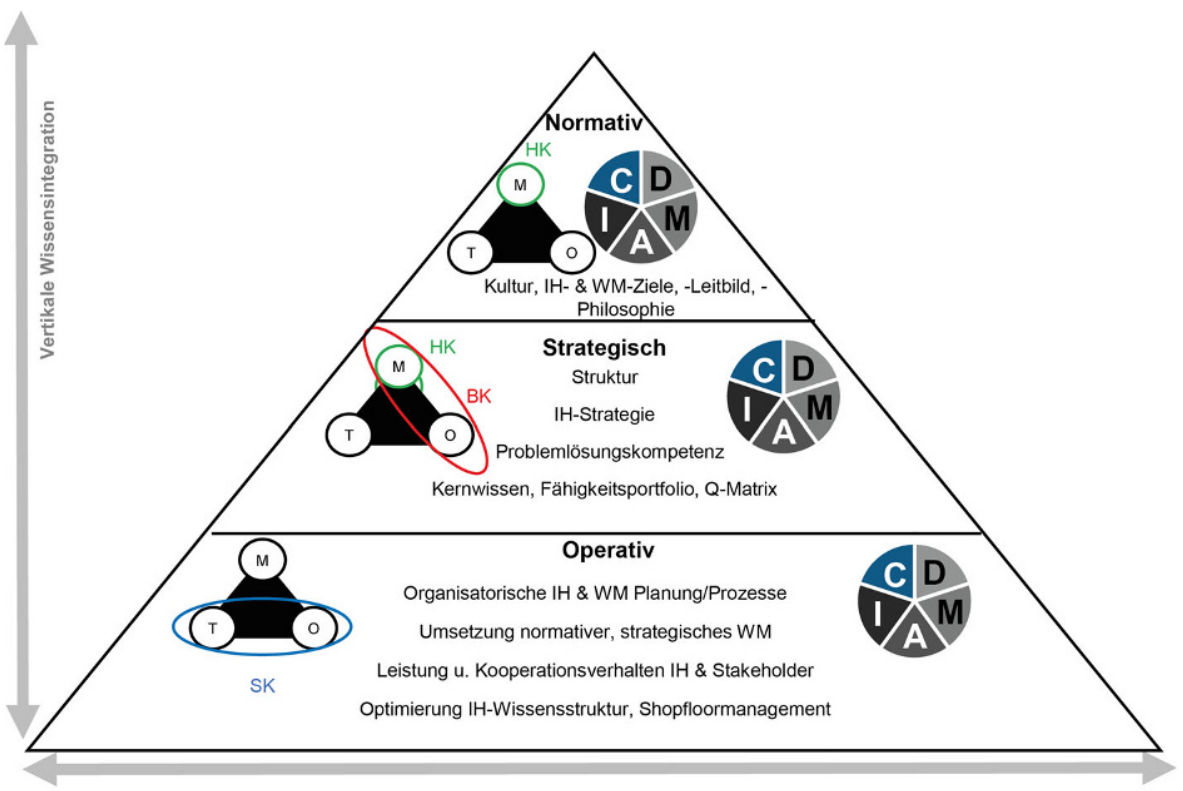

Horizontale Wissensintegration

Abb. 1 Wissensintegration auf den Managementebenen

Nutzung, Wissenspräsentation, Planungs-, Organisations-, Bewertungsmethoden und -systemen. Roehls (2002) Klassifizierung adressiert die Bereiche Infrastruktur, und raum-, kommunikations-, problemlösungs-, arbeits-, personenbezogene Instrumente. Abb. 2 zeigt ein mögliches Klassifizierungsschema von Methoden und Instrumenten des Wissensmanagements für die Anwendung im Bereich Instandhaltung.

Aufbauend auf den 4-Phasen: Identifikation, Erwerb \& Entwicklung, Verteilung $\&$ Nutzung und Bewertung, sind jeweils unterstützende Instrumente definiert worden. Als Grundlage wurde der Regelkreis der Instandhaltung aus der Lean Smart Maintenance Philosophie herangezogen wodurch die normative, strategische und operative Managementebene integriert wird. Der Regelkreis der Instandhaltung beginnt mit der Identifikation der Unternehmens- und Instandhaltungspolitik, die von den vorherrschenden Betriebsbedingungen und der Wandlungsbereitschaft der Mitarbeiter und Mitarbeiterinnen beeinflusst wird. Die Zielplanung sowie die Instandhaltungsstrategieplanung sind zentrale Aufgaben der strategischen Ebene und bilden die Grundlage für die operative Instandhaltungsplanung und -durchführung, gefolgt von der Auftragsüberwachung bis hin zur Schwachstellenanalyse. Für all diese Prozessschritte werden unterschiedliche Instandhaltungs- und UnternehmensDaten und -Informationen benötigt. Für die Identifikation von solchen Daten, Informationen und dem benötigten Wissen werden Instrumente für die Bereiche Organisation, Planung und Repräsentation dargestellt. Die Instrumente für die Wissensrepräsentation sind beispielhaft Instrumente für Wissenskarten, wie etwa Wissensquellkarten, Wissenskulturkarten und/oder Gelbe Seiten zu nennen. Die Abbildung von Wissensflüssen ist anhand von Prozessmodellierungen realisierbar, wofür die 


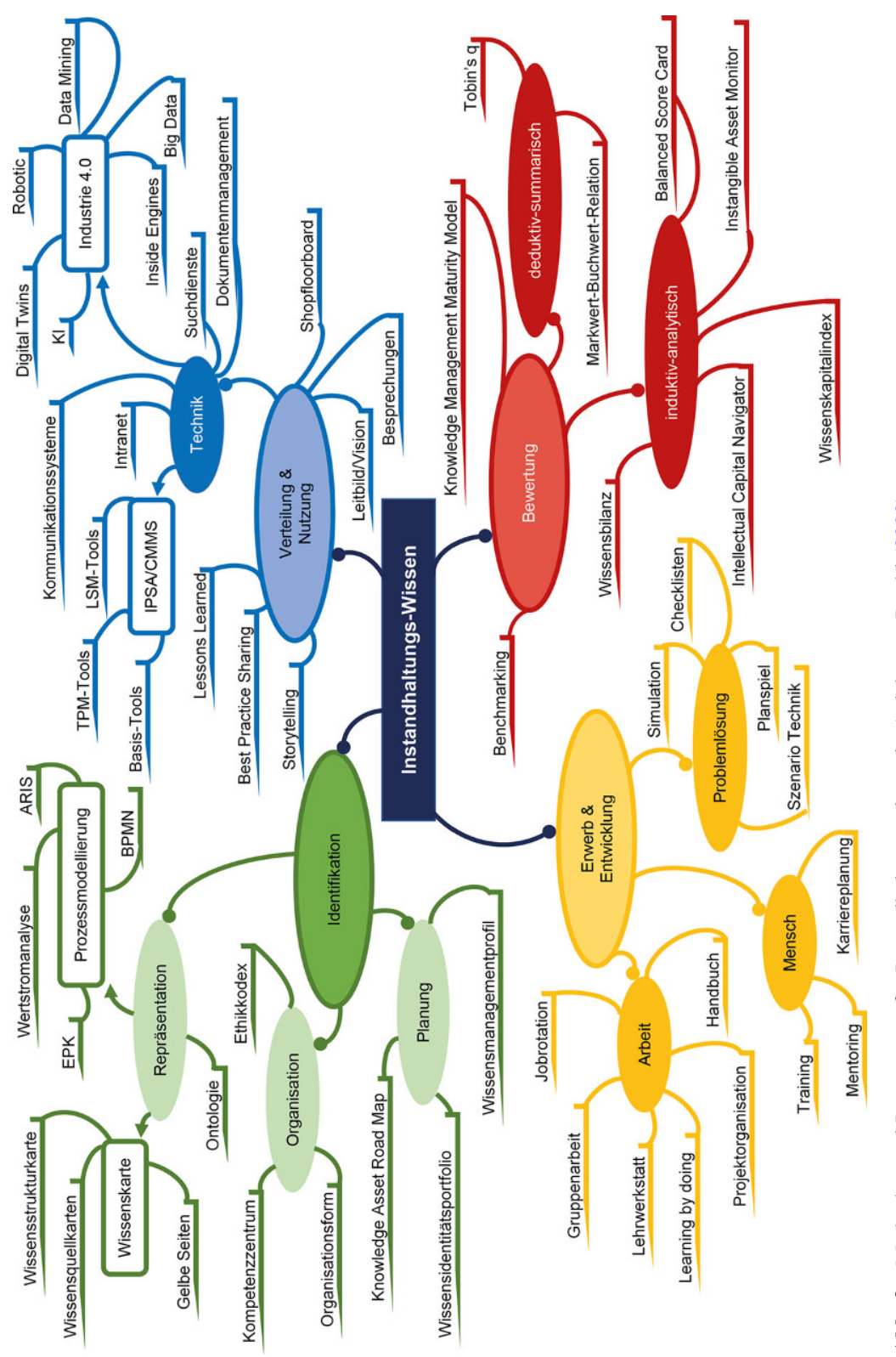

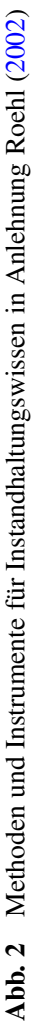

Instrumente EPK (Ereignisgesteuerte Prozesskette), BPMN (Business Process Model and Notation), Wertstromanalyse eingesetzt werden. Die Organisationsform ist für die Wissensidentifikation wichtig, sowie vorhandene Kompetenzzentren. Die Wissensplanung kann mit dem Wissensidentitätsportfolio, Wissensmanagementprofil und einer Knowledge Asset Road Map unterstützt werden. Für den Wissenserwerb und die Wissensentwicklung werden arbeitsbezogene Instrumente einge- 
setzt wie Jobrotation, Lehrwerkstatt, Gruppenarbeit oder Teamarbeit, Learning by doing, Projektorganisation und Handbücher, anhand derer Wissen von bestehenden Instandhaltungsmitarbeitern an weitere Personen vermittelt wird. Durch regelmäßige Trainings, Mentoring und einer Karriereplanung sind personenbezogene Instrumente ebenfalls anzuwenden, um bereits vorhandenes Wissen zu erweitern und neue Anreize für Wissens(weiter)entwicklung zu schaffen. Dafür stehen mitunter verschiedene Problemlösungsinstrumente zur Verfügung wie etwa Planspiel, Checklisten, Simulationen oder Szenario Technik. Das erworbene und (weiter)entwickelte Wissen wird durch verschiedene Instrumente der Informations- und Kommunikationstechnologie verteilt und genutzt, beginnend bei Intranet, Dokumentenmanagementsystemen, bis hin zu Big Data Analytics, Robotik und künstlicher Intelligenz. Zudem sind die Erstellung eines Leitbilds und einer Vision, sowie die Einführung von Lessons Learned, regelmäßigen Besprechungen oder Shopfloorboards adäquate Instrumente für die Wissensnutzung. Benchmarking und ein Knowledge Management Maturity Model bieten die Möglichkeit Wissen zu bewerten, ebenso der Einsatz von deduktivsummarischen Instrumenten wie die Marktwert-Buchwert Relation. Instrumente die vermehrt eingesetzt werden sind induktiv-analytische Instrumente, wie die Balanced Scorecard, der Wissenskapitalindex oder die Wissensbilanz, um Wissen transparenter im Unternehmen zu verankern.

Die steigende Bedeutung von Wissen und dem damit verbundenen notwendigen Aufbaus eines Wissensmanagementsystems spiegelt sich in der gängigen Norm wie der DIN EN ISO 9001:2015 wider. In dieser wurde in der Revision 2015 ein spezielles Augenmerk auf Wissensmanagement gelegt, um sowohl das explizite zu verwalten und $\mathrm{zu}$ verteilen als auch das Bewusstsein über implizites Wissen zu schärfen. Dabei wird darauf Bezug genommen, dass die Instandhaltung gemeinsam mit dem Management das notwendige Wissen, das für die Durchführung und Konformität der Prozesse, Produkte und Dienstleistungen benötigt wird, bestimmen muss. Für die Instandhaltung heißt das, sie legt fest, welche Kompetenzen für die Instandhaltungstätigkeiten benötigt werden.

Dabei wird sowohl das explizite als auch das implizite Wissen, wie etwa Erfahrungen bei Störungen im Hinblick auf Anlagen, Systeme oder Prozesse, berücksichtigt. Hierbei ist der Einsatz von den zuvor genannten Wissensmanagementinstrumenten (Abb. 2) wie Dokumentationen, Lessons Learned mittels einfacher Formulare (Papier oder elektronisch) oder Aufzeichnung in einem IT-System z. B. IPSA (Instandhaltungs-Planung-Steuerungs- und Analyse-System) entscheidend. Die Definition eines strukturierten Dokumentationsprozesses und die Etablierung eines kontinuierlichen Wissensaustauschs zwischen den Mitarbeitern durch regelmäßige Besprechungen, kontinuierliche Lernprozesse und die Anwendung von Problemlösungstechniken ist empfehlenswert. Das explizite Wissen der Instandhaltung bezieht sich auf gespeicherte und verarbeitete Daten von Maschinen oder Anlagen in unterschiedlichster Form wie z. B. Dokumente, Fotos, Aufzeichnungen von Störungen. Dabei ist es wichtig qualitativ hochwertige Daten zu speichern (vollständig, konsistent, regelmäßig, etc.), aus denen alle notwendigen Informationen generiert werden können. Für die Gewährleistung der Vollständigkeit von Daten und Informationen kann am Beginn eine Checkliste zur manuellen Überprüfung erstellt werden. Festgestellte Mängel werden durch die Integration eines kontinuierlichen Verbesserungsprozes- 
ses behoben. Das implizite Wissen, also langjährige Erfahrungen des Instandhaltungspersonals (vgl. Filounek und Steck-Winter 2014) und somit die persönlichen Kompetenzen und Qualifikationen im Unternehmen bilden oftmals die Grundlage vieler instandhaltungsrelevanter Entscheidungen, wie die der IH-Strategiewahl. Die Wahl derselben hängt vor allem von der Datenverfügbarkeit ab. Sind wenig bis keine Anlagendaten vorhanden, ist die Wahl der Instandhaltungsstrategie meist auf Erfahrungswerten basierend. Die Sicherung von implizitem Wissen erfolgt frühzeitig im Unternehmen durch die Integration von Standards im Strategieauswahlprozess. Ist die Datenqualität hingegen sehr hoch und Anlagendaten sind in Echt-Zeit abruf- und analysierbar, sind Erfahrungswerte nur mehr zweitrangig. In diesem Fall steht vor allem das Wissen über die Analyse und Interpretation der Daten und dementsprechende Ableitung von Handlungsempfehlungen im Fokus der Wissensweitergabe. Das Ziel, auch bei der Instandhaltungsstrategiewahl, ist das implizite Wissen soweit als möglich in explizites Wissen zu transferieren. Darüber hinaus wird das vorhandene Wissen der Instandhaltung allen betroffenen Personen langfristig zur Verfügung gestellt und dauerhaft durch den Einsatz von z. B. Wissensmanagementsystemen, Datenbanken, Intranet (vgl. Rahim et al. 2018) bewahrt und Prozesse dadurch optimiert.

Für die Gestaltung des Wissens in einem Unternehmen ist es wesentlich, aufeinander abgestimmte Instrumente und Methoden des Controllings, Wissens- und Informationsmanagements zu verwenden, um eine solide Datenbasis zu schaffen und daraus benötigte Informationen zu erhalten. Einen wichtigen Faktor hierbei spielen neue Technologien, wie Big Data Analytics, Cloud-Computing, Internet der Dinge, Robotik oder künstliche Intelligenz, die einen Wertbeitrag zur Standardisierung und Automatisierung von Prozessen in der Instandhaltung in Kombination mit Wissensmanagement liefern, um den Prozess der Wissenssicherung zu unterstützen bzw. zu erleichtern (vgl. Rahim et al. 2018). Zusätzlich ist es notwendig, dass das ganzheitliche, wertschöpfungsorientierte Wissensmanagement anhand einer strukturierten Vorgehensweise kontinuierlich verbessert wird. Eine systematische Methode bietet das IDEAL (Initiating, Diagnosing, Establishing, Action, Leveraging) Vorgehen aus dem CMM (Capability-Maturity Modell), das als Qualitätsmodell für die Softwareentwicklung gilt und im Standard ISO 15504 enthalten ist. Abgeleitet von IDEAL ist in Abb. 3 eine standardisierte Vorgehensweise in 5-Phasen für eine kontinuierliche Wissensverbesserung (KWV) dargestellt.

In der Initialisierungsphase wird der Projektleiter, das Projektziel und das Projektteam definiert. Die Definition von normativen, strategischen und operativen Wissenszielen im Hinblick auf die zeitliche Umsetzbarkeit ist dabei essentiell, um langfristige strategische und kurz- bis mittelfristige operative Ziele zu setzen. Die Instandhaltungsvision und die instandhaltungskulturellen Aspekte werden über die normativen Wissensziele festgelegt. Operative Wissensziele übersetzen die normativen und strategischen Wissensziele in konkrete Teilziele; ein Beispiel für ein operatives Wissensziel für die Instandhaltung ist „Die internen Experten (Instandhalter) sollen für Kunden (Produktion) besser erreichbar sein. Die akzeptable Antwortzeit ist ..." (vgl. Probst et al. 2006, S. 41 ff). Für die Ableitung strategischer Wissensziele in operative, sind Instrumente des Controllings wie etwa eine Portfolioanalyse anzuwenden. 


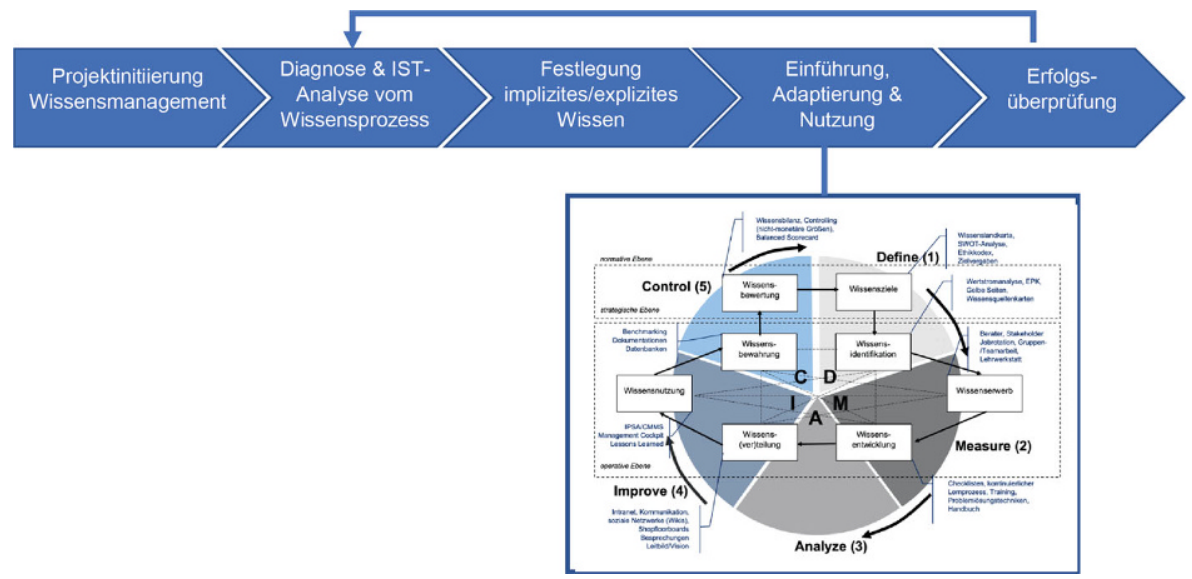

Abb. 3 Vorgehensweise für ein Wissensmanagementprojekt

In der Diagnosephase ist die Sammlung von Dokumenten und Daten über z.B. Anlagen, Arbeitssicherheit, Arbeitsanweisungen, Produktion, für die Instandhaltung notwendig, um einen Überblick über bereits dokumentiertes (explizites) Wissen zu erhalten (vgl. Schneider et al. 2008). Zeitgleich ist die Durchführung von regelmäBigen Workshops erforderlich, um die Instandhaltungsprozesse zu definieren, sowie die vorhandenen Wissensträger zu identifizieren und die notwendigen Informationen für Instandhaltungstätigkeiten festzulegen. Das Ergebnis spiegelt die IST-Situation wider. Daraus ergibt sich ein Überblick über das vorhandene explizite und interne/ externe Wissen und deren Wissensträgern. Zusätzlich müssen messbare Ziele für die Erfolgskontrolle definiert werden.

Zur Messung des Erfolgs von Wissensmanagement in der Instandhaltung, werden bei der Erfolgsüberprüfung, unterschiedliche Kennzahlen herangezogen, aus denen eine Wissensbilanz erstellt werden kann. Zusätzlich gibt es Instrumente wie die BSC (Balanced Score Card) oder der Skandia-Navigator, die eingesetzt werden für die Analyse und Darstellung des immateriellen Vermögens durch verschiedene Bewertungsmethoden (vgl. Sulanjaku 2014). Der umfangreichste und wichtigste Abschnitt des Prozesses ist die Einführung, Adaptierung und Nutzung von Instandhaltungswissen. Dabei sind ein geregelter Ablauf und Transparenz sehr wichtig, um Akzeptanz bei den betroffenen Mitarbeitern und Mitarbeiterinnen zu bekommen. Im nächsten Abschnitt ist solch ein Vorgehen im Detail anhand eines Beispiels dargestellt.

\section{Nachhaltige Wissenssicherung durch gezielten IT-Einsatz in der Instandhaltung}

In der Einführungs-, Adaptierungs- und Nutzungsphase neuer Technologien zur nachhaltigen Wissenssicherung kann ein standardisierter Problemlösungszyklus, wie der PDCA-Zyklus (Plan, Do, Check, Act) oder DMAIC-Zyklus (Define, Measure, Analyze, Improve, Control) angewendet werden. Bei näherer Betrachtung der In- 
tegration von Wissensmanagement im DMAIC-Zyklus ist das Ziel in allen fünf Phasen den wissensorientierten Ansatz zu verankern (vgl. Probst et al. 2006), um personenbezogenes Erfahrungswissen langfristig in der Organisation zu sichern und dem Wissensverlust vorzubeugen. Dabei werden der DMAIC-Zyklus und das MOTModell im Bezug zum Strukturmodell auf allen drei Managementebenen (normativ, strategisch, operativ) integriert (Abb. 1).

Für jede Ebene stehen unterschiedliche Instrumente des Wissensmanagements zur Verfügung (Abb. 2), die eine optimale Ausrichtung Mensch, Organisation und Technik unterstützen und anhand des DMAIC-Zyklus angewendet werden. Für die effiziente und effektive Umsetzung ist die Einbettung der Wissensbausteine nach Probst et al. (2006) in den DMAIC-Zyklus möglich.

Am Beispiel des Einsatzes von Condition Monitoring für permanente Anlagenüberwachung, wird die praxisnahe Anwendung der Instrumente für das Instandhaltungswissen anhand des DMAIC Zyklus nachfolgend beschrieben. Dabei wurden Instrumente aus der Instandhaltungs-Wissens-Box (Abb. 2) gewählt.

\subsection{Wissensziele und -identifikation am Beispiel des Einsatzes von Condition Monitoring}

In der ersten Phase, der Define-Phase liegt der Fokus des Wissensmanagements auf der Definition von Unternehmens-, Instandhaltungs-, und Wissenszielen, wodurch die strategische Managementebene integriert wird. Im konkreten Beispiel, kann ein Ziel sein, die Anlagenverfügbarkeit auf $>98 \%$ zu setzten.

$$
\text { Anlagenverfügbarkeit }=\frac{\text { Nutzungszeit }}{\text { Nutzungszeit }+ \text { technische Ausfallzeit }}[\%]
$$

Auf der operativen Managementebene wird durch die Wissensidentifikation das benötigte und vorhandene Wissen der Instandhaltung identifiziert und transparent dargestellt z.B. anhand von Wissenskarten, Kompetenzkarten (Skill Maps) oder Gelben Seiten (Yellow Pages). Bei dem genannten Beispiel ist Wissen über den Anlagenzustand durch Verwendung von Condition Monitoring Systemen und die damit verbundene Sensorik relevant. Dabei muss geprüft werden, welches Wissen bereits innerhalb der Instandhaltung vorhanden ist bzw. wo noch Lücken vorhanden sind. Dabei kann der Einsatz von Kompetenzkarten hilfreich sein, durch die interne Wissensträger identifiziert werden, wie z. B. die zuständigen Personen für Datenanalytik eines Unternehmens. Des Weiteren sind die Daten-, Informations- und Wissensflüsse zu identifizieren, durch den Einsatz von Prozessmodellierungsmethoden wie z.B. EPK oder einer Wertstromanalyse. Dabei kann der Daten- und Informationsfluss dezidiert dargestellt und Probleme bei der Wissensweitergabe einfach festgestellt werden. 


\subsection{Wissensererb und -entwicklung am Beispiel des Einsatzes von Condition Monitoring}

Der Übergang zum Wissenserwerb und dadurch zur zweiten DMAIC-Phase ist fließend, da der Fokus dieses Prozessschrittes auf der zusätzlichen Erschließung von externen Wissens- und Informationsquellen, wie z.B. Berater, Kooperationen mit Stakeholdern, gelegt ist (vgl. Probst et al. 2006). Im Bereich Anlagenüberwachung durch Sensorik ist die Rekrutierung eines externen Beraters und/oder eines Spezialisten realisierbar, um z.B. die passenden Sensoren bei einer Anlage funktional zu errichten und die Anbindung an ein Condition Monitoring System umzusetzen. Die Trainings für die Mitarbeiter und Mitarbeiterinnen, um mit dem Condition Monitoring System arbeiten zu können, ist durchzuführen. Einzelne Fertigkeiten werden in einer Lehrwerkstätte oder durch Jobrotation erlernt. Externe Schulungen oder Seminare zählen ebenfalls zum Wissenserwerb, sowie Kooperationen mit Kunden, Lieferanten, Mittbewerbern (vgl. Filounek und Steck-Winter 2014, S. 48). In der MeasurePhase wird die Zielerreichung überprüft und im nächsten Schritt Abweichungen im Detail analysiert, indem überprüft wird, ob das bereits vorhandene Wissen über den Einsatz von Condition Monitoring ausreichend ist oder Wissenslücken existieren. Dafür können interne Befragungen, Checklisten, Arbeitssimulationen oder ein Quiz, das alle betroffenen Mitarbeiter und Mitarbeiterinnen durchführen, verwendet werden, um mittels Auswertung der Antworten Wissensdefizite zu erkennen. Daraus werden Erkenntnisse gewonnen, für eine zielgerichtete Verfassung von Handbüchern, Arbeitsanweisung oder Prozessbeschreibungen, zur Verteilung und Nutzung an die betroffenen Personenkreise.

\subsection{Wissensverteilung und -nutzung am Beispiel des Einsatzes von Condition Monitoring}

In Phase 3 (Analyse) wird vor allem auf die Informationsgenerierung und Qualitätsverbesserung bestehender Informationen Wert gelegt, mittels interner Kommunikation, Storytelling (Geschichten erzählen) und regelmäßigen Besprechungen. Die partizipative Entwicklung eines Leitbilds bzw. einer Vision, als Orientierungshilfe und zur Erhöhung der Mitarbeiteridentifikation, sind als konkrete Maßnahmen zu nennen. Für die Verteilung des Wissens ist die Errichtung eines Shopfloorboards wesentlich, wodurch die notwendigen Informationen, z. B. Kennzahlen wie Anlagenverfügbarkeit, die Anzahl an Störungen oder der aktuelle Anlagenzustand, den Mitarbeitern visualisiert werden.

Mit verschiedenen Technologien, wie Intranet, virtuellen Teams, sozialen Netzwerken, werden neue und bestehende Informationen und daraus resultierendes Wissen dem relevanten Personenkreis zur Verfügung gestellt (vgl. Probst et al. 2006). Die Sensordaten, die in das Condition Monitoring System transferiert werden, können direkt über Schnittstellen in ein bestehendes IPSA-System integriert werden. Zusätzlich besteht die Option angemessene Schulungen für die korrekte Anwendung des Condition Monitoring Systems durch externe und/oder interne Lehrbeauftrage für einen definierten Personenkreis in regelmäßigen Abständen durchzuführen, um Wissenslücken zu dezimieren. 
In der vierten, der Improve-Phase, werden Maßnahmen z. B. zur besseren Anwendung von Condition Monitoring Systemen oder Verbesserung der Sensorik abgeleitet, um zukünftig Zielabweichungen einzuschränken und diesen vorzubeugen (vgl. Probst et al. 2006, S. 30). Ein Instrument, das dafür eingesetzt wird, ist die Erstellung von Lessons-Learned-Berichten, in denen Verbesserungsvorschläge für zukünftige Projekte und/oder Tätigkeiten vermerkt werden, sowie bereits aufgetretene Probleme. Ein wichtiger Bestandteil der Improve-Phase ist die Sicherstellung der Wissensnutzung durch den Einsatz von Assistenzsystemen, Info-Center, Dokumentationen, Management Cockpits etc. (vgl. Probst et al. 2006), durch die z. B. das Thema Verbesserung der Anlagenverfügbarkeit mit Condition Monitoring Systemen und Sensorik den Mitarbeitern transparent dargestellt wird. Die Sicherstellung der Wissensnutzung ist anhand von organisatorischen Maßnahmen möglich, wie etwa regelmäßigen Besprechungen. Der Einsatz von Best Practice Beispielen ist eine gute Möglichkeit, Mitarbeitern zu zeigen, wie z.B. Condition Monitoring bei Anlagen im besten Fall anzuwenden sind.

\subsection{Wissensbewahrung und -bewertung am Beispiel des Einsatzes von Condition Monitoring}

Der Einsatz von Datenbanken ist für die Wissensbewahrung hilfreich (vgl. Probst et al. 2006). Dabei werden Daten und Informationen über Probleme bei dem Einsatz von Condition Monitoring Systemen gespeichert und verwaltet. Zusätzlich können Erfahrungswerte (implizites Wissen) einzelner Mitarbeiter und Mitarbeiterinnen schriftlich verfasst, gesammelt und bewahrt werden, damit Fehler, wie z.B. Fehlinterpretation von Störsignalen, zukünftig vermieden werden und explizites Wissen ausgebaut wird. Das Instrument Benchmarking bietet die Möglichkeit der direkten Gegenüberstellung der eigenen Leistungen zu der anderer Unternehmen, um Leistungslücken in einzelnen Bereichen zum Klassenbesten aufzuzeigen. Somit werden Unterschiede und Verbesserungsmöglichkeiten aufgezeigt, sowie neue Erkenntnisse und Ideen gesammelt.

Um den Zyklus abzurunden wird in der Control-Phase die Wirksamkeit gesetzter Maßnahmen anhand der Wissensbewertung überprüft. Dadurch können frühzeitig anlagenverbessernde Maßnahmen implementiert werden. Es werden Kennzahlen verwendet, die als Basis für die Erstellung einer Wissensbilanz dienen, wodurch die Zielerreichung dokumentiert, aber auch eine Erfassung, Bewertung und Verwaltung des intellektuellen Kapitals veranschaulicht wird (vgl. Biedermann 2003) und zur kontinuierlichen Steuerung der Prozesse beiträgt. Im Bereich der Anlagenüberwachung können Kennzahlen wie z.B. Anzahl Sensoren, Anlagenverfügbarkeit, Anzahl Störungen, MTTR (Mean Time To Repair) oder MTBF (Mean Time To Failure) verwendet und durch Soll/Ist-Vergleiche gemessen werden. Aus diesem Grund hat dieser Prozessschritt einen entscheidenden Einfluss auf die zukünftige IHund Wissenszielformulierung. Somit werden Abweichungen frühzeitig identifiziert und Maßnahmen eingeleitet, um den zukünftigen Wissenserhalt zu gewährleisten. Zusätzlich ist es möglich Schwachstellen von Prozessen in den Bereichen der Instandhaltung und dem Wissensmanagement zu verifizieren und diese langfristig zu 
beseitigen. Die Wissenssicherung ist von großer Bedeutung, die anhand von Mitarbeiterqualifikationen und Weiterbildungen realisiert wird.

\section{Zusammenfassung und Ausblick}

Auf Grund des technologischen Fortschritts, der Digitalisierung und Big Data, sollte das organisationale Wissen transparent und verständlich abgebildet werden. Das LSM-Konzept bietet ein gutes Rahmenwerk, um die Instandhaltung effizient und effektiv weiterzuentwickeln (vgl. Passath und Huber 2019; vgl. Biedermann 2016). Die Optimierung der Wissensprozesse anhand des definierten 5-Phasenmodells KWV (Kontinuierliche Wissensverbesserung) mit Einbeziehung von Problemlösungstechniken kann zur Zielerreichung einer wissens-, lern- und wertschöpfungsorientierten Instandhaltung im Sinne eines ganzheitlichen Asset Management Systems und unter Berücksichtigung der Anforderungen der ISO 9001:2015 beitragen. Die Einbeziehung des Faktors Mensch und damit einhergehend die Externalisierung von individuellem, implizitem Wissen führt zugleich zur Eliminierung von Prozessfehlern und Stärkung der Wettbewerbsfähigkeit. Die Verknüpfung der drei Managementebenen und des Strukturmodells (HK, BK, SK) bieten die Möglichkeit Wissen über alle Ebenen hinweg vertikal und horizontal in die Wertschöpfungskette zu integrieren. Folgend ist die frühzeitige Vorbereitung der Mitarbeiter und Mitarbeiterinnen durch gezielte Qualifizierungsmaßnahmen und Weiterbildungen, auf die Transformation durch die Digitalisierung von Bedeutung und sollte forciert werden. Um dieses Potenzial auszuschöpfen, muss auch ein Changemanagementprozess frühzeitig implementiert werden, damit alle Akteure von Beginn an über die Änderungsprozesse informiert werden. Für die Aufwand und Nutzen Betrachtung einzelner Maßnahmen bieten sich Modelle und Methoden wie die Wissensbilanz an, mit denen auch Vergleichswerte über längere Zeiträume abbildbar sind. Gerade Wissensmanagement ist ein langfristiger Prozess, dessen Nutzen erst zu einem späteren Zeitpunkt ersichtlich wird und in Zeiten steigender Digitalisierung nicht außer Acht gelassen werden darf.

Funding Open access funding provided by Montanuniversität Leoben.

Open Access Dieser Artikel wird unter der Creative Commons Namensnennung 4.0 International Lizenz veröffentlicht, welche die Nutzung, Vervielfältigung, Bearbeitung, Verbreitung und Wiedergabe in jeglichem Medium und Format erlaubt, sofern Sie den/die ursprünglichen Autor(en) und die Quelle ordnungsgemäß nennen, einen Link zur Creative Commons Lizenz beifügen und angeben, ob Änderungen vorgenommen wurden.

Die in diesem Artikel enthaltenen Bilder und sonstiges Drittmaterial unterliegen ebenfalls der genannten Creative Commons Lizenz, sofern sich aus der Abbildungslegende nichts anderes ergibt. Sofern das betreffende Material nicht unter der genannten Creative Commons Lizenz steht und die betreffende Handlung nicht nach gesetzlichen Vorschriften erlaubt ist, ist für die oben aufgeführten Weiterverwendungen des Materials die Einwilligung des jeweiligen Rechteinhabers einzuholen.

Weitere Details zur Lizenz entnehmen Sie bitte der Lizenzinformation auf http://creativecommons.org/ licenses/by/4.0/deed.de. 


\section{Literatur}

Biedermann H (2003) Wissensbilanz als Strategie- und Steuerungselement. In: Mathler K, Pechlaner H, Renzl B (Hrsg) Werte schaffen, Perspektiven einer stakeholderorientierten Unternehmensführung. Gabler, Wiesbaden, S 481-498 (Sonderdruck)

Biedermann H (2016) Lean Smart Maintenance: Wertschöpfende, lernorientierte und ressourceneffiziente Instandhaltung. In: Biedermann H (Hrsg) Lean Smart Maintenance: Konzepte. Instandhaltungsforum. TÜV Media, Köln, S 19-29

Biedermann H (2018) Benchmark - Instandhaltung; Eine Studie zum Reifegrad von Instandhaltungsorganisationen der DACH-Region. TÜV Media, Köln

Bullinger H-J, Wörner K, Prieto J (1998) Wissensmanagement - Modelle und Strategien für die Praxis. In: Bürgel HD (Hrsg) Wissensmanagement - Schritte zum intelligenten Unternehmen. Springer, Berlin, S 31-39

Filounek A, Steck-Winter H (2014) Wissensmanagement bei der Instandhaltung von Thermoprozessanlagen. Gaswärme Int 3:45-53

Gronau N (2009) Wissen prozessorientiert managen - Methoden und Werkzeuge für die Nutzung des Wettbewerbsfaktors Wissen in Unternehmen. Oldenbourg Wissenschaftsverlag, München

Lehner F (2006) Wissensmanagement: Grundlagen, Methoden und technische Unterstützung. Hanser, München

Nanoka I, Takeuchi H (1995) The Knowledge-Creating Company: How Japanese companies create the dynamics of innovation. Oxford University Press, New York

Nissen V, Seifert H, Blumstein M (2018) Entwicklung einer Methode zur Unterstützung der Technologieauswahl für die Virtualisierung von Consultingleistungen. HMD 55:801-828

North K, Brandner A, Steininger T (2016) Wissensmanagement für Qualitätsmanager - Erfüllung der Anforderungen nach ISO 9001:2015. Springer, Wiesbaden

Passath T, Huber C (2019) Dynamische Instandhaltungsstrategieanpassung durch Anlagenkritikalitätsbewertung. Berg Huettenmaenn Monatsh 164:7-12. https://doi.org/10.1007/s00501-018-0812-y

Probst G, Raub S, Romhardt K (2006) Wissen managen: wie Unternehmen ihre wertvollste Ressource optimal nutzen, 5. Aufl. Springer Gabler, Wiesbaden

Rahim MA, Stolipin J, Wenzel S (2018) Wissensmanagement in der Produktionssystemplanung. Z Wirtsch Fabrikbetr 113:381-385

Roehl H (2002) Organisationen des Wissens, Anleitung zur Gestaltung. J. G. Cotta’sche, Stuttgart

Schneider G, Geiger IK, Scheuring J (2008) Prozess- und Qualitätsmanagement, Grundlagen der Prozessgestaltung und Qualitätsverbesserung mit zahlreichen Beispielen, Repetitionsfragen und Antworten. Compendio Bildungsmedien AG, Zürich

Seghezzi H, Fahrni F, Herrmann F (2007) Integriertes Qualitätsmanagement: der St. Gallner Ansatz, 3. Aufl. Hanser, München

Sulanjaku M (2014) The contribution of Skandia navigator in intangibles measurements. Int J Econ Commer Manag 2(11):1-10

Urbach N, Ahlemann F (2017) Die IT-Organisation im Wandel: Implikation der Digitalisierung für das ITManagement. HMD 54:300-312 